\title{
Multimedia computer-based learning: a developing role in teaching, CPD and patient care
}

\author{
Chris Williams \& Patrick Harkin
}

Since the 1970s, there has been a progressive increase in the use of computers as a teaching resource. The purpose of this article is to summarise the current uses of computer-based learning (CBL) packages and to highlight how to assess whether a particular multimedia package meets your own requirements for training.

\section{What is a multimedia computer?}

Almost all new computers on the market feature (or can be upgraded to feature) multimedia options such as CD-ROM drives, sound-cards and speakers. They allow sound (such as speech or music) to be played and still or moving pictures (videos) to be shown. This offers particular advantages for psychiatric teaching because the symptoms and signs relevant to psychiatry are assessed at interview.

The power of $\mathrm{CBL}$ is that it allows the presentation of clinical cases and information in an interactive way. Packages should be as interactive as possible to allow the user to seek information and to make judgements and decisions about what further information to seek. See Fig. 1 for an example screen from a psychiatric multimedia product teaching the assessment and management of anxiety.

\section{Effective learning using multimedia}

Different people have different preferred learning styles (Flett, 1996). Some may have a serialist approach and prefer a serial or linear delivery of material; others have a more global/holistic approach to learning with a readiness to link new information to previous experiences and consider different aspects of a problem. Multimedia applications can allow different learning styles to be used flexibly at different points in the package. If, however, only one learning style is used - which may be popular with certain users - it may differentially restrict interest, apparent relevance and learning by users who do not share this learning style.

Examples of different interactive learning styles used include:

(a) Observing a video - for example of a clinical assessment (observing assessment including the way in which screening questions are asked) or of an anxiety management technique being taught to a patient (observing management, e.g. of how to teach diaphragmatic breathing techniques).

(b) Practising a clinical skill - for example the process of mental state examination may be taught by splitting the task into sequential tasks which are completed in a structured

Christopher Williams is a senior lecturer in psychiatry and is the undergraduate psychiatry course organiser at the University of Leeds (Division of Psychiatry and Behavioural Sciences in Relation to Medicine, Level 5, Clinical Sciences Building, St James's University Hospital, Leeds LS9 TTF). He is joint author of a number of interactive computer-based training modules for health care workers and students and of a cognitive-behavioural therapy computer-based self-help package for use by patients. Patrick Harkin is a lecturer in pathology and Head of the Interactive Medical Education Resource at the University of Leeds (Division of Clinical Sciences, Medical Teaching Centre, School of Medicine, University of Leeds, Leeds LS2 9JT). He is also an experienced computer programmer with over 15 years' experience of developing computer-based learning materials. 


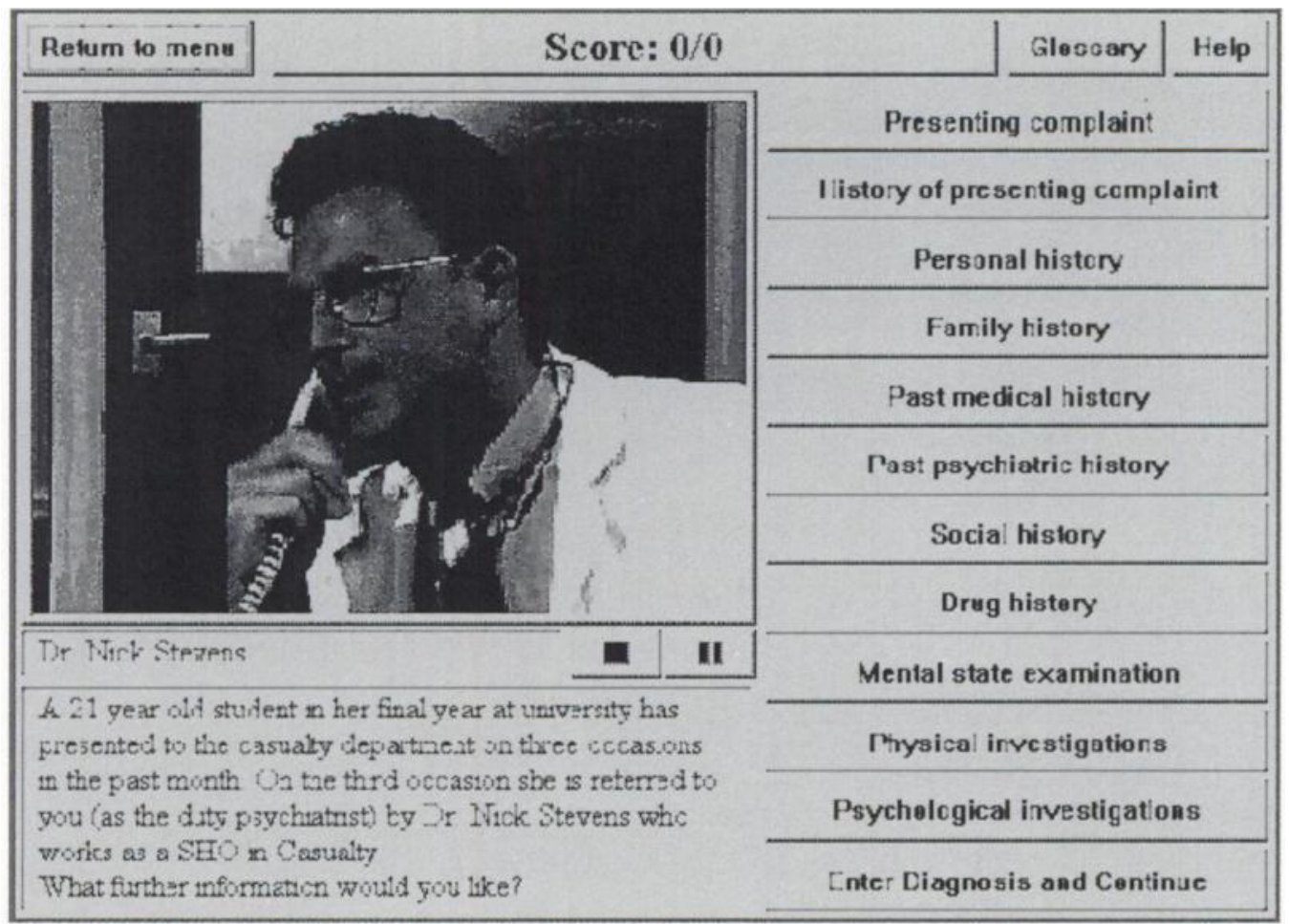

Fig. 1 Example screen from a psychiatric multimedia product teaching the assessment and management of anxiety (Williams et al, 1999)

manner. For example, the appearance and behaviour of a patient can be assessed by requiring the user to make specific responses to questions based on a short video clip (e.g. Is their appearance neat, dishevelled, etc?). Further video clips allow the user to rate the speech flow and form, presence or absence of delusions or hallucinations, and so on. Standardised questions such as the Present State Examination (Wing et al, 1974) may be used to illustrate the process of using sequences of questions to identify mental state symptoms. This can be particularly useful for users new to psychiatry.

(c) Making a diagnosis - the user is asked to make a diagnosis based on all the information (video,

Box 1. Advantages of computer-based learning

Teaching skills to those new in psychiatry

Effective form of CPD

Reliable assessment of clinical skills

Multimedia programmes can be used at the time and place the user chooses

Access to psychiatric teaching for nonpsychiatrists text-based information showing past psychiatric history, the mental state examination, results of physical and psychological tests, etc.) available to them. They can be given specific feedback as to whether their response is correct, and the reasons for supporting or refuting each diagnosis in turn. See Fig. 2 for an example screen.

\section{Advantages and disadvantages of multimedia packages}

\section{Advantages (Box 1)}

\section{Teaching skills to those new to psychiatry}

Many senior house officers new to psychiatry find themselves initially over-faced by the range of unfamiliar terminology and are uncertain about how to take a psychiatric history. Opportunities to sit and watch a senior psychiatrist carry out such assessments are often reduced when qualified. Multimedia techniques allows the creation of a 'patient in a box', which can allow trainees to practise the assessment of common clinical conditions. Multimedia material 


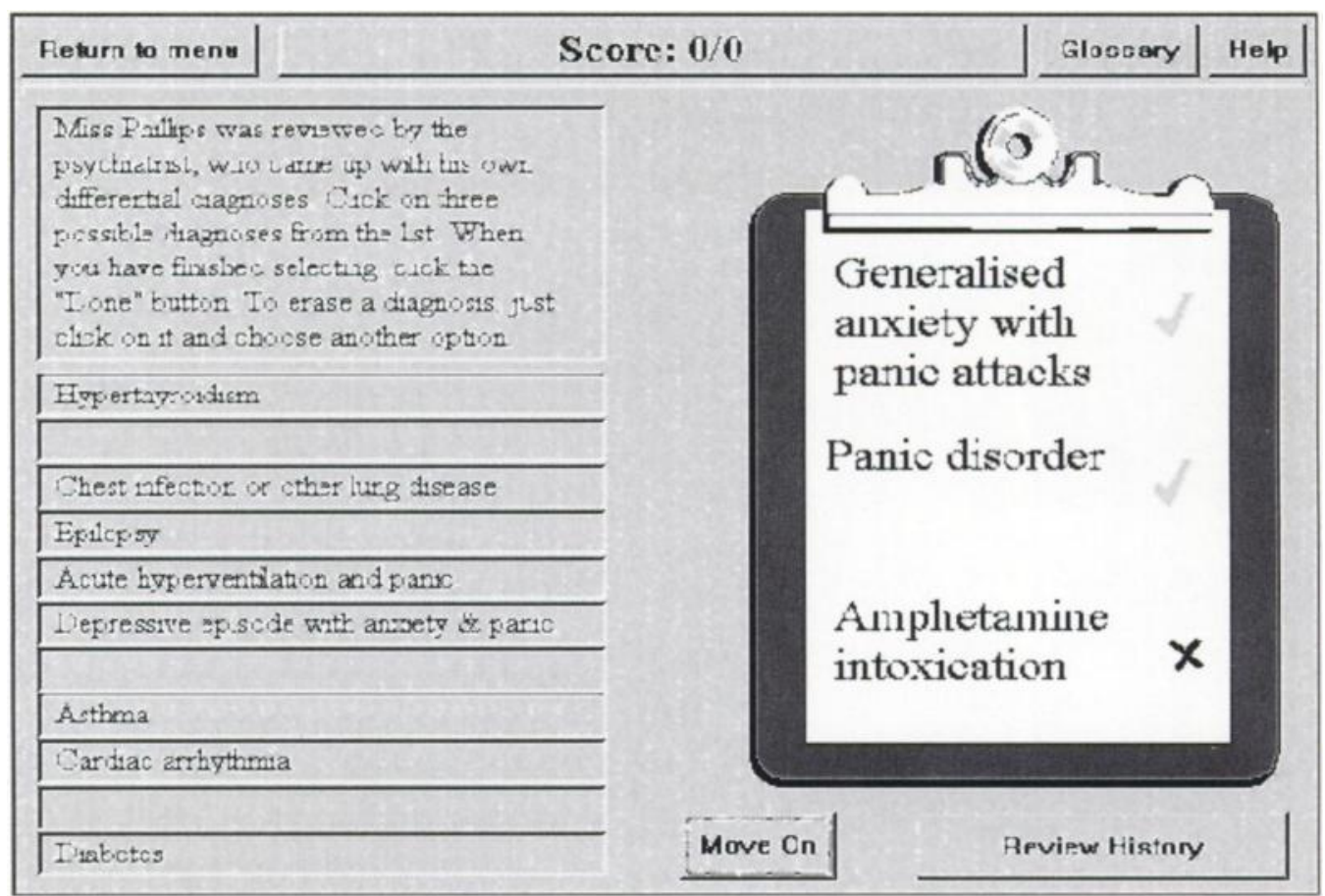

Fig. 2 Example screen showing feedback (Williams et al, 1999) (see Fig. 1)

may be used alone or in supervised sessions to form the basis for discussion between senior trainers and trainees.

\section{Effective form of Continuing Professional Development (CPD)}

Packages may have a useful role in CPD by allowing experienced practitioners to update themselves on recent advances in core and specialist areas of practice. In particular, relatively rare or atypical clinical presentations may be studied. Packages may also have a role in skills maintenance (e.g. to maintain knowledge and skills in a psychiatric subspeciality when one is not normally working in this area).

\section{Reliable assessment of clinical skills}

Multimedia packages allow the presentation, content and marking of case materials to be standardised and thus of greater use in assessment. They can help users assess their knowledge and skills against the 'gold standard' which is hopefully present within the packages.

Multimedia programs can be used at the time and place the user chooses

They allow learning to occur at the time the user wants and at convenient locations such as the office, the library or at home.
Access to psychiatric teaching for non-psychiatrists

Multimedia programs allow quality teaching materials produced by specialists in the field to be available to a wide audience both inside and outside the field of psychiatry.

\section{Disadvantages (Box 2)}

\section{Cost}

Although programming costs have fallen and multimedia machines are increasingly available, many multimedia applications are significantly more expensive than buying a good textbook.

Box 2. Disdvantages of computer-based learning

Cost

Content may easily date

Few packages have been formally peerreviewed

Few are evaluated for thier effectiveness as teaching tools

Few are web-based or use the World Wide Web as an information resource 


\section{Content may easily date}

As with a book, new developments and knowledge may quickly date the contents of the software. It is best to look for recently released products, or ones which offer updates. Skills-based packages are less likely to date than ones which, for example, review current medication.

\section{Few have been formally peer-reviewed}

The Computers in Teaching Initiative (funded by the UK Higher Education Funding Councils (HEFC)) has for the past 10 years provided a national central resource summarising existing multimedia applications for use within higher education. As of February 1999, it lists only three psychiatric multimedia products. Readers can access this website at http://www.cti.ac.uk/. None of these products has been formally reviewed by users of the centre. The HEFC has recently announced funding for a new Learning and Teaching Support Network - full details are available from http://www.niss.ac.uk / education/hefce/pub99/99_20.html.

Box 3. Check-list for evaluating the usefulness of multimedia teaching packages

Are the basic computer requirements to run the package clearly stated?

Is low-cost or free telephone technical support offered?

Is there effective on-line help?

Are the aims and objectives of the package clear and realistic?

Is the package intuitive and easy to use?

Is an overview navigation map available to show where you are in the program?

Are topics and tasks clearly and sequentially structured?

Are individualised responses offered based on user response?

Are a range of teaching styles used which will be valid for the potential user(s)?

Is control placed in the hands of the user?

Are users encouraged to arrive at their own answers or are they spoon-fed?

Are screens uncluttered and the text clear?

Is there an appropriate use of photographs that complement the text?

Is video used to illustrate aspects that cannot be adequately shown by text?

Are ethical issues (e.g. in patient material) addressed (e.g. by using actors)?
Few are evaluated for their effectiveness as teaching tools

The content and process of all forms of teaching should be assessed in order to deliver evidencebased teaching. Multimedia applications are often not evaluated, however, overall, such packages appear as effective as other methods of teaching (Jelovsek \& Adebonojo, 1993). There is a clear need to carry out evaluation as part of open or randomised controlled studies, and for the results of such evaluations to be published so that resource-efficient teaching can be chosen.

Few are web-based or use the World Wide Web as an information resource

Web-based access, or at least the provision of links to allow the utilisation of the vast information resources on the Web (such as hyperlinks to relevant websites), would seem to be a logical way forward for training resources. The use of the new National Health Service Intranet would be one example where effective multimedia training products could be offered to a wide audience of health care professionals and provide an important training resource.

\section{How to choose and use a multimedia training package}

Certain errors in the development of multimedia training are common and users should watch out for them. A short check-list has been prepared to allow you to quickly evaluate the structure and content of multimedia teaching packages (Box 3).

\section{Practical applications}

Anxiety disorders, schizophrenia and paranoid disorders

A number of psychiatric multimedia packages (University of Leeds Innovations Service, 175 Woodhouse Lane, Leeds LS2 3AR; Williams et al, 1999) have been introduced into the medical libraries and hospital sites in Leeds. These are available for users to complete and evaluate their own knowledge and skills. Multiple choice questionnaires assess knowledge and provide specific feedback for incorrect questions. Feedback questionnaires have been completed by both undergraduate and postgraduate staff to assess acceptability, accuracy of content and ease of use. Packages have been updated as a result. This process of production, 
evaluation and updating provides a model that may be used to improve the functionality and usefulness of such training tools.

\section{The future}

This article has focused on the role of computer packages in improving the knowledge, skills and attitudes of health care workers. Computers may also have a valuable role in educating patients in their own self-management of psychiatric conditions (Marks et al, 1998). Their role in self-treatment has been investigated by a range of studies carried out in the USA and in the UK. With the increasing sophistication of information technology, computer systems have moved from pure instruction-giving to allow individually tailored responses - which was undreamed of even five years ago. The advent of evidence-based psychotherapies - such as cognitive-behavioural therapy (Andrews, 1996) which may be manualised and have a large educational component offers great promise for the development of evidence-based self-management by patients. Such relatively structured self-treatment packages, when coupled with the development of complex interactive multimedia computers, may, in future, come to supplement the individual and group, medical and non-medical treatments offered within the health service today. This offers the opportunity that training materials used by health care trainees and professionals today may develop into the self-management packages used by patients tomorrow. The challenge in both staff and patient education is to seek high-quality product development and to require that such packages have clear aims and objectives and are shown to meet these aims and objectives in evaluative studies.

\section{References}

Andrews, G. (1996) Talk that works: the rise of cognitive behaviour therapy. British Medical Journal, 313, 1501-1502.

Flett, A. (1996) Student Personality and Approaches to Learning. Teaching and Learning Occasional Paper No 1. Leicester: Leicester University.

Jelovsek, F. \& Adebonojo, L. (1993) Learning principles as applied to computer-assisted instruction. MD Computing, 10, 165-172.

Marks. I., Shaw, S. \& Parkin, R. (1998) Computer-aided treatments of mental health problems. Clinical Psychology, Science and Practice, 5, 151-170.

Williams, C. J. , Aubin, S., Harkin, P. J. R., et al (1999) Anxiety Disorders. Leeds: University of Leeds Innovations Service.

Wing, J. K., Cooper, J. E. \& Sartorius, N. (1974). Measurement and Classification of Psychiatric Symptoms. Cambridge: Cambridge University Press.

\section{Multiple choice questions}

1. The following are essential components of multimedia computers:
a CD-ROM
b video-card
c floppy disc
d sound-card.

2. The following are desirable features to consider when choosing a multimedia package:

a a clear statement of whether actors or real patients have been used in any video

b pages full of text and no photographs

c the use of photographs unconnected to the programme content

d video which presents lecture-style information.

3. The creation of CBL teaching material:

a should not be attempted in small departments

b requires certification under national guidelines

c can be undertaken by students

d benefits from a multi-disciplinary team approach.

4. Multimedia packages:

a should be sited in a quiet place such as an open library

b can replace all elements of the undergraduate medical course

c have consistently been shown to be at least as good as other teaching methods

d cannot be shared across a network. 